\title{
Post Chemoradiation PET SUV is highly Predictive of Overall Survival in Esophageal Cancer
}

\author{
H Lomas ${ }^{1}$, SE Hoffe ${ }^{2}$, J Weber ${ }^{3}$, TJ Dilling ${ }^{2}$, MD Chuong ${ }^{2}$, K Almhanna ${ }^{3}$, RC Karl ${ }^{3}$, K. Meredith ${ }^{3}$ and R Shridhar²*
}

${ }^{1}$ University of South Florida School of Medicine, USA

${ }^{2}$ Department of Radiation Oncology, Florida, USA

${ }^{3}$ Gastrointestinal Tumor Program, H. Lee Moffitt Cancer Center \& Research Institute, Tampa, FL, USA

\begin{abstract}
Purpose: Positron Emission Tomography (PET) scan is a vital tool in the staging, prognosis and response evaluation of gastrointestinal malignancies. The purpose of this study is to determine the prognostic significance of PET standardized uptake values (SUV) in esophageal cancer treated with chemoradiotherapy (CRT).

Methods: An IRB approved esophageal cancer database was queried for patients who completed treatment for esophageal cancer with CRT between 2006 and 2010. Patients were included in the analysis if they had nonmetastatic esophageal cancer that completed definitive or preoperative CRT and had pre- and post-CRT PET scans. Patients treated with induction chemotherapy were excluded. The pre-and post-CRT SUV maximum values were obtained. Univariate analysis for overall survival (OS) was performed with Kaplan-Meier and log-rank analysis or hazard ratio model. Multivariate analysis (MVA) for OS was performed with a Cox proportional hazard ratio model.

Results: We identified 77 patients who met inclusion criteria with a median followup of 16 months (range 4 - 43 months). Univariate analysis demonstrated that post-CRT SUV max and percent change SUV max were prognostic for OS, while pre-CRT SUV was not prognostic. In addition, there were no deaths in patients with a post-CRT SUV $\max$ of $\leq 3$. MVA demonstrated that only post-CRT SUV $\max (\mathrm{HR} 1.401 ; 95 \% \mathrm{Cl}: 1.061-1.850)$ was prognostic for OS, while age, gender, surgery, histology, tumor length and stage, were not.

Conclusions: Our series demonstrates that post-CRT maximum SUV was the strongest predictor of survival in esophageal cancer while pre-CRT SUV was not. Percent change SUV max was prognostic on univariate analysis but not on multivariate analysis. Prospective studies to evaluate the role of post-CRT SUV in the management of esophageal cancer are needed.
\end{abstract}

Keywords: Esophageal cancer; Chemoradiation; PET SUV; Survival; Surgery

\section{Introduction}

In the United States, an estimated 17,000 people will be expected to be diagnosed with esophageal cancer in 2011 with nearly 15,000 dying from the disease [1]. Squamous cell carcinoma and adenocarcinoma account for $>90 \%$ of all esophageal cancers. While there has been a steady decline in the incidence of squamous cell carcinomas due to reduction of smoking, a dramatic rise in the incidence of adenocarcinomas has been noted because of increases in obesity. Overall 5-year survival for localized esophageal cancer is $34 \%$. Surgery alone results in very poor outcomes. A recent meta-analysis confirmed a survival benefit to trimodality therapy of neoadjuvant chemoradiation followed by surgical resection [2]

Positron emission tomography (PET) using [18F]fluorodeoxyglucose (FDG) is an imaging modality with a higher sensitivity compared to computed tomography (CT) or endoscopic ultrasound (EUS) for detecting the presence of metastatic disease [3-9]. This has been shown to affect the surgical management of up to $20 \%$ of patients $[3,5,6]$. PET measures tumor metabolic activity reported as a standardized uptake value (SUV) and may potentially be prognostic for survival. A recent meta-analysis of seven studies demonstrated that the higher the initial SUV, the worse the survival [10]. In contrast, Rizk et al. showed no correlation between SUV and survival and also correlated high SUV with better response to therapy [11]. Restaging PET scans after neoadjuvant therapy can detect metastases in $8 \%$ of patients [12]. In addition, PET has also been used to monitor response to induction chemotherapy [13-15] and may determine who may benefit from surgery [16]. However, we have previously shown that a negative post treatment PET scan had a positive predictive value for predicting a pathologic complete response of only $35 \%$ [17]. There are conflicting data as others have shown a limited impact on the prognostic significance of PET $[13,18,19]$. One disadvantage of PET is determining response from treatment-related inflammation that can make interpreting response difficult $[15,20]$. The purpose of this study was to determine the prognostic significance of SUV pre and post chemoradiation therapy (CRT) in esophageal cancer patients who were treated definitively or preoperatively.

\section{Materials and Methods}

\section{Patients}

An IRB-approved esophageal database was queried to identify non-metastatic esophageal cancer patients treated at the H. Lee Moffitt Cancer Center with concurrent chemoradiotherapy (CRT) between

*Corresponding author: Ravi Shridhar, MD, PhD, Department of Radiation Oncology, H. Lee Moffitt Cancer Center \& Research Institute, 12902 Magnolia Drive, Tampa, Florida, USA, Tel: 813-745-3053; Fax: 813-745-7231; E-mail: ravi.shridhar@moffitt.org

Received March 17, 2012; Accepted March 26, 2012; Published March 30, 2012

Citation: Lomas H, Hoffe SE, Weber J, Dilling TJ, Chuong MD, et al. (2012) Post Chemoradiation PET SUV is highly Predictive of Overall Survival in Esophagea Cancer. J Nucl Med Radiat Ther 3:125. doi:10.4172/2155-9619.1000125

Copyright: ( 2012 Lomas H, et al. This is an open-access article distributed under the terms of the Creative Commons Attribution License, which permits unrestricted use, distribution, and reproduction in any medium, provided the original author and source are credited. 
2006 through 2010. Patients were treated curatively with either definitive or preoperative intent and had pre- and post-CRT PET scans that could be analyzed on our AW workstation. Patients were excluded if they underwent induction chemotherapy. Verification of measurements was performed by comparing radiology reports to our measurements of SUV max on the AW workstation and no discrepancies were found. Patient characteristics are shown in Table 1.

\section{Staging}

All patients had biopsy proven adenocarcinoma or squamous cell carcinoma that was verified by specialized GI oncology pathologists. Patients underwent contrast-enhanced computed tomography (CT) of chest and abdomen, PET-CT scans and endoscopic ultrasound. Patients with T1N1 or T2N0 or higher were referred for either definitive or preoperative chemoradiation and were evaluated by a surgeon, medical oncologist and radiation oncologist. Restaging PET and CT scans were ordered 4-8 weeks after completion of CRT. Nonmetastatic patients who passed cardiac and pulmonary clearance were offered surgical resection. Surgery was performed 6-12 weeks after CRT. One patient with T1N0 who had multiple medical comorbidities prohibiting surgery was included in the analysis.

\section{Chemoradiation}

Patients underwent CT-simulation of chest and abdomen after immobilization in a customize Vac-lok (Civco Medical Solutions) cradle. Gross tumor volume (GTV) was delineated by the radiation oncologist either marked by endoscopically place fiducials or treatment planning PET-CT scans that were fused to the treatment-planning CT scan. Patients also underwent 4D-CT scan to assess respiratory motion of the GTV. Internal target volumes (ITVs) of gross disease were generated. A clinical target volume (CTV) encompassing a 3-4

\begin{tabular}{|c|c|}
\hline Demographic & N (\%) \\
\hline Median Age (years)(range) & $61.9(32-81)$ \\
\hline $\begin{array}{l}\text { Gender } \\
\text { Male } \\
\text { Female }\end{array}$ & $\begin{array}{l}64(79.2) \\
13(20.8)\end{array}$ \\
\hline $\begin{array}{l}\text { Histopathology } \\
\text { Adenocarcinoma } \\
\text { Squamous Cell Carcinoma }\end{array}$ & $\begin{array}{l}63(81.8) \\
14(18.2)\end{array}$ \\
\hline $\begin{array}{l}\text { Location } \\
\text { Upper } \\
\text { Middle } \\
\text { Distal } \\
\text { GEJ } \\
\end{array}$ & $\begin{array}{c}4(5.2) \\
3(3.9) \\
32(41.6) \\
38(49.3)\end{array}$ \\
\hline $\begin{array}{l}\text { Surgery } \\
\text { Yes } \\
\text { No }\end{array}$ & $\begin{array}{l}49(63.6) \\
28(36.4)\end{array}$ \\
\hline $\begin{array}{l}\text { AJCC }\left(7^{\text {th }} \text { ed }\right) \text { T-stage } \\
\text { T1 } \\
\text { T2 } \\
\text { T3 } \\
\text { T4 }\end{array}$ & $\begin{array}{c}8(10.4) \\
9(11.7) \\
46(59.7) \\
14(18.2)\end{array}$ \\
\hline $\begin{array}{l}\text { AJCC }\left(7^{\text {th }} \text { ed }\right) \text { N-stage } \\
\text { N0 } \\
\text { N1 } \\
\text { N2 }\end{array}$ & $\begin{array}{c}10(13) \\
43(55.8) \\
24(31.2)\end{array}$ \\
\hline $\begin{array}{l}\text { AJCC }\left(7^{\text {th }} \text { ed }\right) \text { Stage } \\
\text { IA } \\
\text { IB } \\
\text { IIB } \\
\text { IIIA } \\
\text { IIIB } \\
\text { IIIC }\end{array}$ & $\begin{array}{c}1(1.3) \\
4(5.2) \\
20(26) \\
25(32.5) \\
14(18.2) \\
13(16.8)\end{array}$ \\
\hline
\end{tabular}

Table 1: Patient Characteristics. $\mathrm{cm}$ superior margin, 3-4 cm distal margin and 3-5 mm radial margin was contoured. Regional abdominal lymphatics were covered in distal/ GE junction tumor sites. Planning target volumes (PTVs) with margins individualized based on whether daily image guidance was used. IMRT plan constraints consisted of: lung (Mean $<16 \mathrm{~Gy}$, V20 < 30\%, V5 $<60 \%$ ), heart (Mean < 30Gy), spinal cord (Max < 50 Gy), kidneys (Mean $<12 \mathrm{~Gy}$ ) and liver (V30 < 30\%). Concurrent chemotherapy was delivered in all patients with the regimen chosen at the discretion of the medical oncologist. Doses to the CTV ranged from 45-50.4 Gy in 1.8 Gy fractions. Doses to GTV ranged form $45-60$ Gy in 1.8-2 Gy fractions.

\section{FDG Whole-body PET}

Pre- and post-CRT FDG-PET scans were performed. Patients received 10 to $15 \mathrm{mCi}$ of [18F]-FDG after 6 hours of fasting and imaging was performed 1-2 hours after injection. Attenuation correction was performed using low-dose computed tomography. FDG-PET images were interpreted by an experienced nuclear radiologist and correlated with computed tomography. Quantitative analysis was performed using standardized uptake values (SUVs) and calculated as the maximum value after injection. Region of interest analysis were used to calculate the maximal FDG concentration within the primary tumor mass. SUV max values were obtained by correcting for the injected dose and the patient's weight. For the purposes of this study, only 18FDG uptake in the primary site of disease was analyzed.

\section{Statistical analysis}

Univariate analysis for overall survival (OS) was performed with Kaplan-Meier and log-rank analysis or hazard ratio model. Median values were determined for each SUV parameter and used as a cutoff for Kaplan-Meier analysis. Multivariate analysis for OS was performed with a Cox proportional hazard ratio for age, gender, surgery, stage, histology, tumor length, post-CRT SUV max and percent change of SUV max. Similar results were obtained for SUV mean values (data not shown). Statistical analysis was performed with STATA IC (Stata Statistical Software, Release 10.0; Strata Corp, College Station, TX).

\section{Results}

We identified 77 patients who underwent initial and restaging 18F-FDG PET-CT scan prior to definitive or preoperative CRT for esophageal cancer who had all scans performed at Moffitt Cancer Center. The median followup was 16 months (range 4 - 43 months). The median age at initial presentation was 61.9 years (range, 32-81). Sixty-three $(81.8 \%)$ patients of the patients had adenocarcinoma and $14(18.2 \%)$ patients had squamous cell carcinoma. AJCC $7^{\text {th }}$ edition stage I, II, IIIA, IIIB and IIIC disease was present in 5\%, 20\%, 25\%, 14\% and $13 \%$, respectively. The locations of the tumors were $4 \%$ upper, $3 \%$ middle, 32\% distal and 38\% at the gastro-esophageal junction. Surgical resection was performed in $49(63.6 \%)$ patients (Table 1$)$.

Figure 1 illustrates differences in survival by median SUV max for pre-CRT SUV max (median 12.2), post-CRT SUV max (median 4.4) and percentage change in SUV max (median 57\%). Univariate KaplanMeier analysis demonstrated that there were significant differences in survival when accounting for post-CRT SUV max and percentage change in SUV max, while there was no significant difference is survival based on pre-CRT SUV max. For patients with post-CRT SUV $\max$ of $\leq 4.4$ vs $>4.4,2$ year OS was $59 \%$ versus $25.4 \%$, respectively ( $\mathrm{p}$ $=0.0054$ ) (Figure 1A). In patients with percentage change in SUV max of $>57 \%$ versus $\leq 57 \%$, 2 year OS was $86.2 \%$ vs $50.9 \%$, respectively $(\mathrm{p}=0.0069)$ (Figure 1B). In contrast, patients with pre-CRT SUV max 
Citation: Lomas H, Hoffe SE, Weber J, Dilling TJ, Chuong MD, et al. (2012) Post Chemoradiation PET SUV is Highly Predictive of Overall Survival in Esophageal Cancer. J Nucl Med Radiat Ther 3:125. doi:10.4172/2155-9619.1000125

Page 3 of 5
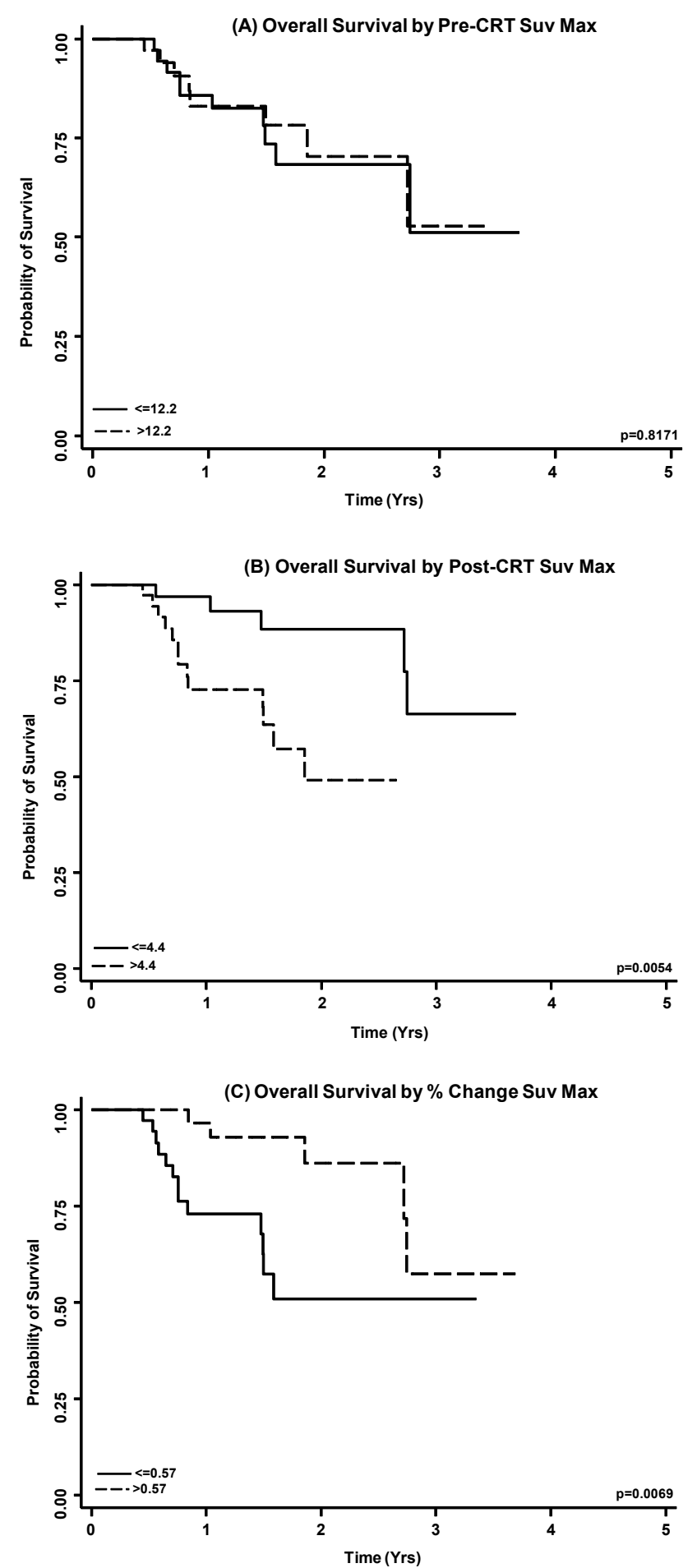

Figure 1: Kaplan-Meier analysis of overall survival based on median SUV values for (A) pre-CRT SUV; (B) post-CRT SUV; (C) percentage change SUV.

of $\leq 12.2$ versus $>12.2,2$ year OS was $34 \%$ versus $52 \%$, respectively ( $\mathrm{p}$ $=0.8171$ ) (Figure 1C).

Table 2 displays hazard ratio (HR) values at various SUV max levels for pre-CRT and post-CRT and at differing percentage differences for relative change SUV max. There is no threshold level that predicts for differences in mortality for pre-CRT SUV max, while $>50 \%$ (HR 0.281 ; 95\% CI: $0.105-0.754 ; \mathrm{p}=0.012)$ and $>60 \%$ (HR 0.285; 95\% CI: 0.100 $-0.809 ; \mathrm{p}=0.018$ ) change in SUV max predict for lower mortality and higher post-CRT SUV max predicts for higher mortality. Interestingly, we could not calculate the HR for post-CRT $\max$ for $\leq 3 \mathrm{vs}>3$ because there were no deaths in patients with a post-CRT SUV $\max$ of $\leq 3$. On multivariate analysis, only higher post-CRT SUV max (HR 1.401; 95\%CI: 1.061- 1.850; $\mathrm{p}=0.017$ ) predicted for worse OS. Age, gender, EUS tumor length, histopathology, surgery, stage and percentage change in SUV were not predictive for survival (Table 3).

\section{Discussion}

FDG PET scans have emerged as a vital tool in the management of esophageal cancers. PET is used for initial staging, preoperative staging, restaging after neoadjuvant therapy and response to treatment. PET has been shown to change the management in $8-20 \%$ of esophageal cancer patients $[3,5,6]$. The results from this study shows that on univariate analysis, a $>50-60 \%$ change in SUV conferred reduced mortality, while higher post-CRT SUV max was associated with higher mortality. In fact, there were no deaths in patients with post-CRT SUV $\max \leq 3$. Pre-

\begin{tabular}{|c|c|c|c|}
\hline Pre-CRT SUV & HR & $95 \% \mathrm{Cl}$ & p-value \\
\hline$\leq 8$ vs $>8$ & 2.062 & $0.597-7.128$ & 0.253 \\
\hline$\leq 10$ vs $>10$ & 1.285 & $0.482-3.425$ & 0.617 \\
\hline$\leq 12$ vs $>12$ & 0.933 & $0.369-2.357$ & 0.883 \\
\hline$\leq 14$ vs $>14$ & 0.625 & $0.223-1.760$ & 0.375 \\
\hline$\leq 16$ vs $>16$ & 0.845 & $0.276-2.580$ & 0.767 \\
\hline$\leq 18$ vs $>18$ & 1.037 & $0.299-3.956$ & 0.955 \\
\hline \% SUV Change & HR & $95 \% \mathrm{Cl}$ & p-value \\
\hline$\leq 30 \%$ vs $>30 \%$ & 1.338 & $0.386-4.637$ & 0.647 \\
\hline$\leq 40 \%$ vs $>40 \%$ & 0.976 & $0.347-2.742$ & 0.963 \\
\hline$\leq 50 \%$ vs $>50 \%$ & 0.281 & $0.105-0.754$ & 0.012 \\
\hline$\leq 60 \%$ vs $>60 \%$ & 0.285 & $0.100-0.809$ & 0.018 \\
\hline$\leq 70 \%$ vs $>70 \%$ & 0.271 & $0.060-1.221$ & 0.089 \\
\hline Post-CRT SUV & HR & $95 \% \mathrm{Cl}$ & p-value \\
\hline$\leq 3$ vs $>3^{*}$ & - & - & - \\
\hline$\leq 4$ vs $>4$ & 6.36 & $1.439-28.11$ & 0.015 \\
\hline$\leq 5$ vs $>5$ & 3.248 & $1.174-8.988$ & 0.023 \\
\hline$\leq 6$ vs $>6$ & 2.76 & $1.026-7.430$ & 0.044 \\
\hline$\leq 7$ vs $>7$ & 4.833 & $1.744-13.394$ & 0.002 \\
\hline
\end{tabular}

*- hazard ratio for 3 could not be calculated since there were no deaths in patients with Post-CRT SUV $\leq 3$; HR - hazard ratio; Cl - confidence interval; CRT chemoradiotherapy; SÜV - standard uptake value

Table 2: Hazard Ratio by PET Parameter.

\begin{tabular}{|c|c|c|c|}
\hline Variable & HR & $\mathbf{9 5 \%}$ Cl & p-value \\
\hline Age $^{*}$ & 0.976 & $0.912-1.041$ & 0.459 \\
\hline Gender (vs. Male) & 1.125 & $0.133-9.532$ & 0.914 \\
\hline Stage III (vs Stage I/II) & 1.974 & $0.344-11.340$ & 0.446 \\
\hline EUS Tumor Length* & 0.436 & $0.922-1.379$ & 0.242 \\
\hline Histopathology (vs SqCC) $^{*}$ & 1.128 & $0.022-8.826$ & 0.589 \\
\hline Surgery (vs. No surgery) $^{*}$ & 0.380 & $0.091-1.582$ & 0.184 \\
\hline \% SUV change* & 0.526 & $0.028-9.977$ & 0.669 \\
\hline Post-CRT SUV* $^{*}$ & 1.401 & $1.061-1.850$ & $\mathbf{0 . 0 1 7}$ \\
\hline
\end{tabular}

* - continuous variable; HR - hazard ratio; Cl - confidence interval; CRT chemoradiotherapy; SUV - standard uptake value; EUS - endoscopic ultrasound; $\mathrm{SqCC}$ - squamous cell carcinoma

Table 3: Multivariate Analysis for Overall Survival. 
CRT SUV max did not impact survival. On multivariate analysis, the only prognostic factor for survival was post-CRT SUV max. Surgery, histopathology, age, gender, stage, tumor length and percentage change in SUV max were not prognostic for survival.

In a previously published series from our institution, 81 patients who had pre- and post-CRT PET scans, underwent neoadjuvant CRT followed by surgical resection were analyzed to determine if a negative PET scan post-CRT predicted for a pathologic complete response. A negative and positive post-CRT PET scan had positive predictive values for predicting pathologic complete response and residual disease of $35 \%$ and $70 \%$, respectively [17]. Despite the fact that a negative PET had a low probability for predicting a pathologic complete response, our analysis shows that only post-CRT SUV max was predictive for OS, while surgery was not. This may be explained by tumor biology in PET-responders who are not pathologic responders. These tumors likely are hypoxic and thus more radioresistant, but remain PETnegative because of their low metabolic activity or very small size. This may explain why surgery in this subset of patients does not impact OS because these tumors may be in a dormant state. These results are supported by the recently published work of Monjazeb et al. [16]. In their analysis of 163 patients treated with CRT, 88 (54\%) underwent surgical resection. Patients with negative PET (defined as SUV $<3$ ) had improved 2 year OS ( $71 \%$ versus $11 \%$; $<0.01)$. In addition, they also reported that patients who achieve a negative PET do not benefit from surgical resection.

Our analysis revealed that pre-CRT SUV was not prognostic for survival. This is in agreement with Rizk et al. who showed that initial SUV did not predict for survival in 189 esophageal cancer patients. Additionally, they showed that lower SUV correlated with poorer response to CRT resulting in lower pathologic complete response rates and higher rates of residual nodal disease [11]. In contrast, a metaanalysis of seven studies demonstrated that the higher the SUV, the worse the survival (HR 1.86) [10]. Many of the data included in the analysis was from older studies and the authors of this study admit to lack of access to many specifics which may have impacted their results.

PET has been used to monitor response to treatment as well [13-15,20]. Weber et al. studied 40 patients treated with induction chemotherapy with a PET scan performed 14 days after baseline. A cutoff value of $35 \%$ change in PET SUV predicted for survival ( $p=$ 0.04 ) [20]. Weider et al. correlated tumor response to patient survival in esophageal cancer based on PET response to one cycle of chemotherapy. After baseline PET scan, 38 patients were treated with induction platinum-based multiagent chemotherapy and then underwent PET 14 days later. A $>40 \%$ decrease in SUV correlated with increased survival [15]. This data led to the MUNICON trial [14]. In this prospective trial, 119 patients with distal esophageal or gastroesophageal junction were treated with 2 weeks of platinum and fluorouracil chemotherapy regimen followed by a 14 day PET scan. Responders were defined as an SUV decrease of $35 \%$. Responders continued to receive neoadjuvant chemotherapy for 12 weeks and then underwent resection. Nonresponders discontinued chemotherapy and proceeded directly to surgery. While median OS was not reached in responders, median OS was 25.8 months (19.4-32.2) in non-responders (HR 2.13 [1.14-3.99], $\mathrm{p}=0.015)$. An ongoing CALGB trial is using PET to identify patients who need modification of their ongoing treatment (clinicaltrial. gov; NCT01333033). In arm 1, patients receive modified FOLFOX-6 therapy comprising oxaliplatin IV over 2 hours and leucovorin calcium IV over 2 hours on day 1 and fluorouracil IV continuously on days 1-5. Treatment repeats every 14 days for 3 courses. Patients then undergo PET/CT scan. Patients with responsive disease (tumor metabolic activity decreased by $\geq 35 \%$ ) receive 3 additional courses of FOLFOX-6 therapy and undergo concurrent radiotherapy (RT) (3D-conformal or intensity-modulated) once daily, 5 days a week, for approximately 6 weeks. Patients without responsive disease (tumor metabolic activity did not decrease by $35 \%$ ) cross over to arm 2 during RT. In arm 2, Patients receive carboplatin IV over 30 minutes and paclitaxel IV over 1 hour on days 1 and 8 . Treatment repeats every 21 days for 2 courses. Patients then undergo PET/CT scan. Patients with responsive disease (tumor metabolic activity decreases $\geq 35 \%$ ) continue to receive carboplatin IV over 30 minutes and paclitaxel IV over 1 hour once weekly for 5 weeks and undergo RT (3D-conformal or intensity-modulated) once a day, 5 days a week, for approximately 6 weeks. Patients without responsive disease (metabolic activity did not decrease by $35 \%$ ) cross over to arm 1 during RT.

The question remains as to which modality is best for assessing response to CRT in esophageal cancer, PET, EUS or surgery. We previously showed that response directly correlates with survival [21]. Meredith et al. showed that patients achieving pCR had 5-year disease-free survival (DFS) and overall survival (OS) of 52\% and 52\%, respectively, compared with $36 \%$ and $38 \%$ in pPR and $22 \%$ and $19 \%$ in NR $(\mathrm{P}<0.0001, \mathrm{P}<0.0001)$. Additionally, we showed that a negative and positive post-CRT PET scan had positive predictive values for predicting pathologic complete response and residual disease of 35\% and $70 \%$, respectively [17]. Ngamruengphong et al. examined 7 studies of 966 patients to determine the diagnostic accuracy of FDG-PET and EUS [22]. The sensitivity of EUS and FDG-PET ranged from 20 to $100 \%$ and 42 to $100 \%$, respectively. The specificity ranged from 36 to $100 \%$ and 27 to $100 \%$, respectively. The areas under the curve were 0.86 (95\% confidence interval [CI]: 0.77-0.96) for EUS and 0.80 (95\% CI: 0.72-0.89) for FDG PET $(\mathrm{P}=0.37)$. The maximum joint sensitivity and specificity $\left(\mathrm{Q}^{*}\right.$ index) values for EUS and FDG-PET were 0.79 (95\% CI: $0.70-0.88)$ and 0.74 (95\% CI: $0.66-0.81)$, respectively $(\mathrm{P}=0.38)$. There was no difference in accuracy between early FDG-PET and FDG-PET after completion of neoadjuvant therapy. EUS and FDG-PET have similar overall diagnostic accuracy for assessment of response to neoadjuvant therapy in patients with esophageal cancer.

One disadvantage of PET is determining response from treatmentrelated inflammation that can make interpreting response difficult $[15,20]$. In our series, however, we found an extremely low rate of esophagitis on restaging PET. We believe this is likely due to the improved techniques of IMRT as a modality in esophageal cancer and the timing of restaging PET (4-6 weeks).

One weakness of this study is that is retrospective but hypothesis generating. It does add to the growing literature on the prognostic significance of PET in esophageal cancer. The role of adjuvant chemotherapy in esophageal cancer remains controversial. A prospective trial evaluating the role of adjuvant chemotherapy based on post-CRT SUV may help to determine who will benefit from adjuvant chemotherapy.

\section{Conclusion}

Our series demonstrates that percentage change of SUV and postCRT maximum SUV were prognostic for survival in esophageal cancer on univariate analysis, however, only post-CRT SUV was prognostic for survival on multivariate analysis. Patients in this series did not benefit from surgery. Multi-center prospective trials to evaluate the role of postCRT SUV in the management of esophageal cancer to determine the need for surgery, adjuvant chemotherapy, or surveillance are needed. 
Citation: Lomas H, Hoffe SE, Weber J, Dilling TJ, Chuong MD, et al. (2012) Post Chemoradiation PET SUV is Highly Predictive of Overall Survival in Esophageal Cancer. J Nucl Med Radiat Ther 3:125. doi:10.4172/2155-9619.1000125

\section{References}

1. Siegel R, Ward E, Brawley O, Jemal A (2011) Cancer statistics, 2011: the impact of eliminating socioeconomic and racial disparities on premature cancer deaths. CA Cancer J Clin 61: 212-236.

2. Sjoquist KM, Burmeister BH, Smithers BM, Zalcberg JR, Simes RJ, et al. (2011) Survival after neoadjuvant chemotherapy or chemoradiotherapy for resectable oesophageal carcinoma: an updated meta-analysis. Lancet Oncol 12: 681-692.

3. Block MI, Patterson GA, Sundaresan RS, Bailey MS, Flanagan FL, et al. (1997) Improvement in staging of esophageal cancer with the addition of positron emission tomography. Ann Thorac Surg 64: 770-776

4. Flamen P, Lerut A, Van Cutsem E, De Wever W, Peeters M, et al. (2000) Utility of positron emission tomography for the staging of patients with potentially operable esophageal carcinoma. J Clin Oncol 18: 3202-3210.

5. Flanagan FL, Dehdashti F, Siegel BA, Trask DD, Sundaresan SR, et al. (1997) Staging of esophageal cancer with 18F-fluorodeoxyglucose positron emission tomography. AJR Am J Roentgenol 168: 417-424.

6. Luketich JD, Friedman DM, Weigel TL, Meehan MA, Keenan RJ, et al. (1999) Evaluation of distant metastases in esophageal cancer: 100 consecutive positron emission tomography scans. Ann Thorac Surg 68: 1133-1136.

7. Meyers BF, Downey RJ, Decker PA, Keenan RJ, Siegel BA, et al. (2007) The utility of positron emission tomography in staging of potentially operable carcinoma of the thoracic esophagus: results of the American College of Surgeons Oncology Group Z0060 trial. J Thorac Cardiovasc Surg 133: 738 745.

8. Rasanen JV, Sihvo El, Knuuti MJ, Minn HR, Luostarinen ME, et al. (2003) Prospective analysis of accuracy of positron emission tomography, computed tomography, and endoscopic ultrasonography in staging of adenocarcinoma of the esophagus and the esophagogastric junction. Ann Surg Oncol 10: 954-960.

9. van Vliet EP, Heijenbrok-Kal MH, Hunink MG, Kuipers EJ, Siersema PD (2008) Staging investigations for oesophageal cancer: a meta-analysis. $\mathrm{Br} \mathrm{J}$ Cance 98: 547-557.

10. Pan L, Gu P, Huang G, Xue H, Wu S (2009) Prognostic significance of SUV on PET/CT in patients with esophageal cancer: a systematic review and metaanalysis. Eur J Gastroenterol Hepatol 21: 1008-1015.

11. Rizk NP, Tang L, Adusumilli PS, Bains MS, Akhurst TJ, et al. (2009) Predictive value of initial PET-SUVmax in patients with locally advanced esophageal and gastroesophageal junction adenocarcinoma. J Thorac Oncol 4: 875-879.

12. Bruzzi JF, Swisher SG, Truong MT, Munden RF, Hofstetter WL, et al. (2007)
Detection of interval distant metastases: clinical utility of integrated CT-PET imaging in patients with esophageal carcinoma after neoadjuvant therapy Cancer 109: 125-134.

13. Downey RJ, Akhurst T, Ilson D, Ginsberg R, Bains MS, et al. (2003) Whole body 18FDG-PET and the response of esophageal cancer to induction therapy: results of a prospective trial. J Clin Oncol 21: 428-432.

14. Lordick F, Ott K, Krause BJ, Weber WA, Becker K, et al. (2007) PET to assess early metabolic response and to guide treatment of adenocarcinoma of the oesophagogastric junction: the MUNICON phase II trial. Lancet Oncol 8: 797 805

15. Wieder HA, Brücher BL, Zimmermann F, Becker K, Lordick F, et al. (2004) Time course of tumor metabolic activity during chemoradiotherapy of esophageal squamous cell carcinoma and response to treatment. J Clin Oncol 22: 900-908.

16. Monjazeb AM, Riedlinger G, Aklilu M, Geisinger KR, Mishra G, et al. (2010) Outcomes of patients with esophageal cancer staged with [(1) F]fluorodeoxyglucose positron emission tomography (FDG-PET): can postchemoradiotherapy FDG-PET predict the utility of resection? J Clin Oncol 28: 4714-4721.

17. McLoughlin JM, Melis M, Siegel EM, Dean EM, Weber JM, et al. (2008) Are patients with esophageal cancer who become PET negative after neoadjuvan chemoradiation free of cancer? J Am Coll Surg 206: 879-886.

18. Konski AA, Cheng JD, Goldberg M, Li T, Maurer A, et al. (2007) Correlation of molecular response as measured by 18-FDG positron emission tomography with outcome after chemoradiotherapy in patients with esophageal carcinoma. Int J Radiat Oncol Biol Phys 69: 358-363.

19. Vallböhmer D, Hölscher AH, Dietlein M, Bollschweiler E, Baldus SE, et al. (2009) [18F]-Fluorodeoxyglucose-positron emission tomography for the assessment of histopathologic response and prognosis after completion of neoadjuvant chemoradiation in esophageal cancer. Ann Surg 250: 888-894.

20. Weber WA, Ott K, Becker K, Dittler HJ, Helmberger H, et al. (2001) Prediction of response to preoperative chemotherapy in adenocarcinomas of the esophagogastric junction by metabolic imaging. J Clin Oncol 19: 3058-3065

21. Meredith KL, Weber JM, Turaga KK, Siegel EM, McLoughlin, et al. (2010) Pathologic response after neoadjuvant therapy is the major determinant of survival in patients with esophageal cancer. Ann Surg Oncol 17: 1159-1167.

22. Ngamruengphong S, Sharma VK, Nguyen B, Das A (2010) Assessment of response to neoadjuvant therapy in esophageal cancer: an updated systematic review of diagnostic accuracy of endoscopic ultrasonography and fluorodeoxyglucose positron emission tomography. Dis Esophagus 23: 216 231. 